\title{
Reduction of Adult Intussusception: More Harm Than Benefit
}

\author{
Pankaj Kumar Garg ${ }^{1,2} \cdot$ Bhupendra Kumar Jain ${ }^{1}$
}

Published online: 14 April 2015

(C) Société Internationale de Chirurgie 2015

Dear Sir,

We read the article titled "adult intussusception: a retrospective review" by Honjo et al. [1] with great interest. Based on their experience of 44 patients of adult intussusception, the authors [1] concluded: (a) preoperative diagnosis is possible in all patients with currently available radiological modalities; and (b) reduction of intussusception, preoperative or intraoperative, offers many advantages and so should be the standard approach in the management of adult intussusception. Based on our experience of nine patients of adult intussusception [2,3], we agree with the authors partly. With the current advancements in radiological modalities, preoperative diagnosis of intussusception can be arrived safely in almost all patients. Contrast-enhanced computed tomography (CT) of abdomen is the most sensitive method to confirm bowel intussusception [4].

The optimal treatment of adult intussusception is controversial. The reported case series by Honjo et al [1] had 24 malignant lesions in 41 patients (about $60 \%$ ). The authors have not described postoperative complications of surgery in the series; the risk of anastomotic leak increases if the edematous bowel is involved in the anastomosis. We believe that preoperative/intraoperative reduction should not be attempted in adult intussusception as contrast to

Pankaj Kumar Garg

dr.pankajgarg@gmail.com

1 Department of Surgery, University College of Medical Sciences and Guru Teg Bahadur Hospital, University of Delhi, Delhi 110095, India

2 Department of Surgical Oncology, Dr BRA Institute Research Cancer Hospital, All India Institute of Medical Sciences, New Delhi 110029, India pediatric patients where reduction is the standard practice. Potential surgical concerns regarding the reduction in adult intussusception are (a) high frequency of associated malignancy in adult intussusception strongly favors primary en bloc resection to avoid perforation and tumor seedling (b) high frequency of associated bowel gangrene prohibits handling of friable and edematous bowel as it may cause dissemination of bacteria and anastomotic complications [2]. The concern to avoid resection of excessive length of bowel can be taken care by applying gentle traction on the ileum entering the intussusception. This maneuver yields considerable length of normal looking ileum without causing disruption/ complete reduction of the intussussception and associated complications. Needless to say, we also believe that there cannot be a substitute for surgeon's wisdom to individualize the surgical treatment in a given patient based on intraoperative findings.

\section{References}

1. Honjo H, Mike M, Kusanagi H, Kano N (2015) Adult intussusception: a retrospective review. World J Surg. 39:134-138. doi:10. 1007/s00268-014-2759-9

2. Prasad D, Mohanty D, Garg PK, Agarwal V, Jain BK (2011) Adult intussusception: is associated bowel gangrene common? Trop Gastroenterol. 32:45-49

3. Garg PK (2010) Idiopathic adult intussusception. Saudi J Gastroenterol. 16:305-306

4. Mrak K (2014) Uncommon conditions in surgical oncology: acute abdomen caused by ileocolic intussusception. J Gastrointest Oncol. 5:E75-E79 